\title{
The Fetal Visual Evoked Potential
}

\author{
JAMES R. WOODS, JR. AND MARK A. PLESSINGER \\ Department of Obstetrics and Gynecology, University of Cincinnati Medical Center and the A. E. Seeds Perinatal \\ Research Center, University of Cincinnati, Cincinnati, Ohio 45267-0526
}

\begin{abstract}
Five singleton fetal lambs were surgically instrumented at 110-121 days gestation (term gestation = 145 days) for measurement of visual evoked potentials (VEP) in utero. Light flashes were generated by a small incandescent bulb secured under the right fetal eyelids and the visual responses were recorded through stainless steel electrodes implanted in the fetal scalp. Four of the five fetal lambs were surgically instrumented between 110-113 days. VEPs recorded in these four animals from 111-114 days exhibited $N_{1}, P_{1}$, and $N_{2}$, but lacked the $P_{2}$ component. All VEP components $\left(N_{1}, P_{1}, N_{2}, P_{2}\right)$ were measureable by 115-118 days in these fetuses. One fetus was surgically instrumented at 121 days and VEPs containing all components were measurable until 125 days gestation when the equipment dislodged in utero. Serial VEP recordings from all five fetal lambs from 111-128 days indicated no change in latency to $N_{1}$ while significant latency changes occurred in $P_{1}, N_{2}$, and $P_{2}$ suggesting maturational development of the visual system. Histological assessment of the eyes from three animals at delivery revealed only thickening and increased vascularity of the cornea in response to the light source resting on the fetal eye. In conclusion, these results suggest that the fetal visual evoked potential has application in the longitudinal study of normal visual development in utero and offers a technique for studying visual response changes to adverse perinatal events. (Pediatr Res 20: 351-355, 1986)
\end{abstract}

\section{Abbreviation}

VEP, visual evoked potential

The VEP has been used to measure visual function and development in the premature human newborn $(1,2)$, term human newborn $(3-5)$, and term newborn lambs $(6,7)$. In these studies, the VEP components, consisting of an initial negative deflection $N_{1}$, a positive deflection $P_{1}$, a second negative deflection $\mathrm{N}_{2}$, and a large positive deflection $\mathrm{P}_{2}$, exhibit shortening of latencies with advancing neonatal age. These observations suggest maturation within the visual system is occurring during the newborn period.

Recently, the visual evoked potential of the exteriorized nearterm fetal lamb has been shown to be similar to VEPs obtained from term newborn lambs (6). The presence of VEPs from exteriorized fetal lambs indicates that the visual system is functional prior to birth and develops during fetal life. Assessment of visual responsiveness in this fetal model however, is limited by the short amount of recording time and the stress imposed upon the fetal lamb during the process of surgical exteriorization and

Received September 24, 1985; accepted November 20, 1985.

Correspondence should be sent to James R. Woods, Jr., M.D., Department of Obstetrics and Gynecology. University of Rochester School of Medicine and Dentistry, 601 Elmwood Ave., Rochester, NY 14642.

Supported by the Whittaker Foundation, Camp Hill, PA instrumentation. Measurement of the fetal VEP in the chronically instrumented fetal lamb offers the opportunity for longitudinal assessment of visual development in utero. The results presented here 1) describe the procedures for obtaining visual evoked potentials from the chronically instrumented fetal lamb; 2) illustrate the typical VEPs obtained; and 3) present latency measurements of the VEPs from 111 to 128 days gestation.

\section{METHODS}

Five singleton lambs were surgically instrumented between $110-121$ days gestation (term gestation $=145$ days) for measurement of fetal VEPs. After a spinal anesthetic (Pontocaine) was administered to the pregnant ewe, a midline abdominal incision was made to expose the uterus. A fetal forelimb was then palpated and delivered through a small incision in the uterus. Following administration of subdermal injection of local anesthesia ( $2 \%$ Xylocaine), a polyvinyl catheter was inserted into the brachial artery for arterial blood gas measurements. The incision was then closed, the fetal forelimb placed back in the uterus, and the uterine incision closed. All subsequent fetal instrumentation was also carried out following administration of subdermal anesthesia ( $2 \%$ Xylocaine). A second incision was then made in the uterus and the vertex of the fetal head exposed. A stainless steel wire encased in polyvinyl tubing was placed subdermally at the vertex of the fetal head and secured with 3-0 silk to serve as the active electrode. A similar stainless steel wire electrode was placed subdermally anterior to the right fetal ear to serve as the reference electrode. Through the same uterine incision, the fetal head was repositioned and the fetal eye ipsilateral to the reference electrode was exposed for placement of the light source for visual stimulation. The fetal eyelids at this stage of gestation are sealed shut and require manipulation to open.

The light source consists of a miniature incandescent bulb sealed in silicone rubber (Fig. 1). During construction of the light source, this unit is secured to a hard contact lens while the silicone rubber hardens. The contact lens is then carefully removed leaving a smooth, pliable surface which rests on the surface of the fetal eye. A curved polyflex arm, approximately 6 $\mathrm{cm}$ across, is secured $2 \mathrm{~cm}$ behind the light source and acts to prevent rotation of the light source during fetal movement. The incandescent bulb is connected to a shielded two-wire cable and encased in polyvinyl tubing.

Following administration of local anesthesia to the eyelids, the light is placed on the cornea of the fetal eye between the eyelids and is secured by sutures to the fetal eyelids for stability. The ends of the polyflex arm are sutured to the fetal skin to provide additional support. The fetal head is placed back into the uterus and the uterine incision closed. The stainless steel wires, light source wires, and arterial catheter are then brought out through the maternal abdominal incision to a pouch on the side of the ewe.

Four of the five fetuses were surgically instrumented between 110-113 days gestation in order to obtain visual responses in this earlier period of gestation. The fifth fetus was surgically 


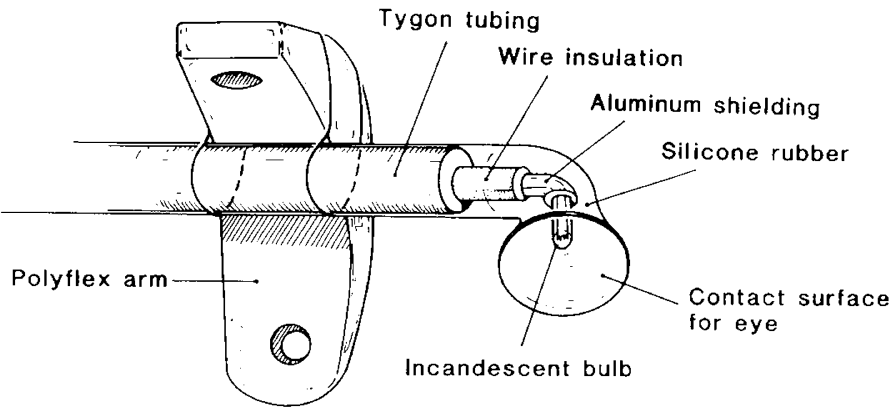

Fig. 1. Artist's sketch of the light source used to elicit fetal visual evoked potentials in utero.

instrumented at 121 days but VEP measurements were only obtained at 123 days due to dislodgement of the stainless steel electrodes at 125 days gestation.

Two of the five fetal lambs died in utero at 120 and 121 days gestation. Three fetal lambs were delivered at 133,136, and 140 days by cesarean section. Immediately following delivery, these newborn lambs were given a lethal dose of Soccumb. One newborn lamb was tested for VEPs before sacrifice. The instrumented eye and the uninstrumented eye of all three animals were removed in order to assess corneal damage from the light source resting on the fetal eye during the 4 to 18 days of in utero testing.

Immediately upon removal, the eyes were fixed in $10 \%$ buffered formalin before examination by the Eye Pathology Laboratory of the University of Cincinnati. After gross examination, the eyeballs were opened and the corneas were removed. After dehydration in alcohol and clearing with chloroform, the corneas were impregnated and embedded in paraffin. Corneal slices $8-\mu$ thick were fixed to slides with egg albumen. After hematoxylin periodic-acid Schiff, Phloxine, and Safranin O stains were applied, microscopic examination and analysis were performed by the Pathology Laboratory.

Measurement of fetal VEPS. All fetal lambs were tested for VEPS beginning 1 day after surgery and repeated every other day. On each day of VEP testing, a $0.5-\mathrm{ml}$ arterial blood sample was collected anaerobically in a 1.0 - $\mathrm{ml}$ glass syringe and analyzed for fetal blood $\mathrm{pH}, \mathrm{pCO}_{2}$, and arterial $\mathrm{pO}_{2}$.

Light flashes were triggered and the VEP responses were averaged by a Synap I (Infant Hearing Assessment Foundation, San Francisco, CA) Signal Averaging Computer. Square wave pulses of $100 \mathrm{~V}$ and of $10 \mathrm{~ms}$ duration were generated by a Grass S-9 stimulator (Grass Industries, Quincy, MA) and delivered to a $28 \mathrm{~V}$ incandescent bulb (CM6838, General Instrument, Chicago, IL) producing a candlepower of 23.56 lumens per flash. Thirty-five light flashes were presented at a rate of one every $2 \mathrm{~s}$ and a 400-ms response interval following each flash was recorded. The visual responses were averaged by the Synap I unit with a 1 to $30 \mathrm{~Hz}$ bandpass frequency and a $2 \mathrm{~ms}$ per point sampling interval. The final averaged signal was recorded on $2 \frac{1}{4}$ inch wide heat-sensitive paper with a 400 -ms grid. All recordings were done in triplicate to assure reproducibility.

The fetal visual evoked potentials were analyzed by determining the latency period in milliseconds (stimulus to individual wave deflections) to the first major negative or upward deflection, designated $\mathrm{N}_{1}$, the first positive deflection, or $P_{1}$, the second negative deflection, $\mathrm{N}_{2}$ and the second and most pronounced positive wave deflection. $\mathrm{P}_{2}$. Three recording runs from each fetus on a given day of testing were used for latency measurements. Tabular data represents the average of all individual recording runs used in latency measurements. For graphic data, numerical latency values of the three consecutive VEP recordings from a single animal on a given day of recording are averaged together for each data point. From these serial measurements, linear regression analysis with test for statistical significance using
Pearson's correlation was performed on the graphic data. Amplitude measurements were not presented in this study because of high variability between recordings.

\section{RESULTS}

Reproducible VEP measurements containing all four major wave components, $\mathrm{N}_{1}, \mathrm{P}_{1}, \mathrm{~N}_{2}$, and $\mathrm{P}_{2}$, were obtained from all five fetuses tested. Figure 2 illustrates a typical fetal visual evoked potential from a 128-day-old fetus and the newborn VEP recorded when the same fetus was delivered by cesarean section at 140 days. In this example, the similar configurations of the fetal and newborn VEP and the major deflections, $N_{1}, P_{1}, N_{2}$, and $P_{2}$ can be seen. Analysis of fetal arterial blood samples for blood gas determinations were as follows: $\mathrm{pH}=7.35 \pm .04 ; \mathrm{pCO}_{2}=$ $38.8 \pm 2.7 ; \mathrm{pO}_{2}=20.3 \pm 4.0$ (data presented as mean $\pm 1 \mathrm{SD}$ ).

The VEPs recorded from the four fetal lambs which were surgically instrumented between 110-113 days and tested between 111-114 days contained the $N_{1}, P_{1}$, and $N_{2}$ components but lacked the major $\mathrm{P}_{2}$ component seen in the later gestational age fetus and newborn. In these animals, however, all VEP components were measurable by $115-118$ days.

Representative examples of VEP recordings from each of five fetuses are shown in Figure 3. These VEP examples were recorded from the five fetuses from 117 to 128 days. In each example the overlays of three consecutive recordings of VEPs are shown. The major wave deflections, $\mathrm{N}_{1}, \mathrm{P}_{1}, \mathrm{~N}_{2}$, and $\mathrm{P}_{2}$ are indicated.

One of the fetuses (fetus 4, Fig. 3) tested exhibited VEPs from 114 to 128 days. Electrode dislodgement, indicated by high electrode impedance, occurred after 128 days, and prevented further VEP recordings until 140 days gestation when the lamb was delivered by cesarean section and retested for VEPs as a neonate. Figure 4 illustrates a series of VEP recordings obtained from this fetal lamb. On the 1st day of testing at 112 days gestation, no reproducible VEPs were noted. At 114 days gestation, the first recorded VEP contained the early components, $\mathrm{N}_{1}$, $\mathrm{P}_{1}$, and $\mathrm{N}_{2}$ with no evidence of the late positive deflection, $\mathrm{P}_{2}$. This late positive deflection first appears at 116 days gestation, occurring at approximately $300 \mathrm{~ms}$. With serial testing in this

FETAL VEP

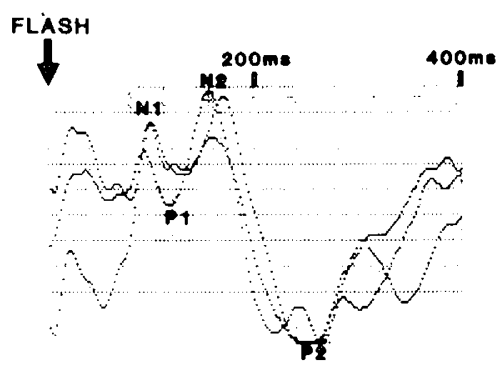

ONE DAY

NEWBORN VEP

(140 DAYS GESTATION)

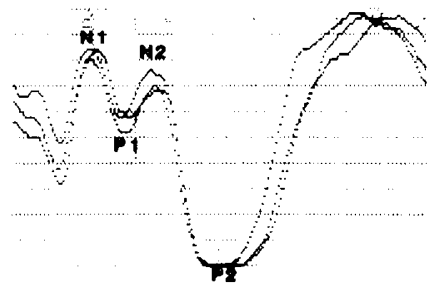

EACH TRACING REPRESENTS THE OVERLAY

OF THREE AVERAGED RESPONSES

TO 35 LIGHT FLASHES

Fig. 2. Typical fetal and newborn lamb VEPs. The major wave deflections $\mathrm{N}_{1}, \mathrm{P}_{1}, \mathrm{~N}_{2}$, and $\mathrm{P}_{2}$ are identified. 
FETUS \# 1

117 DAYS

FETUS \# 2

119 DAYS

FETUS \# 3

123 DAYS

FETUS \#4

126 DAYS

FETUS \# 5

128 DAYS
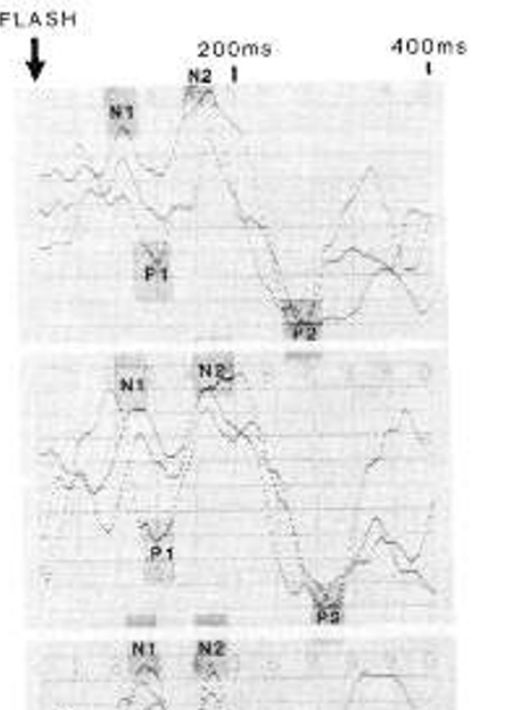
S 


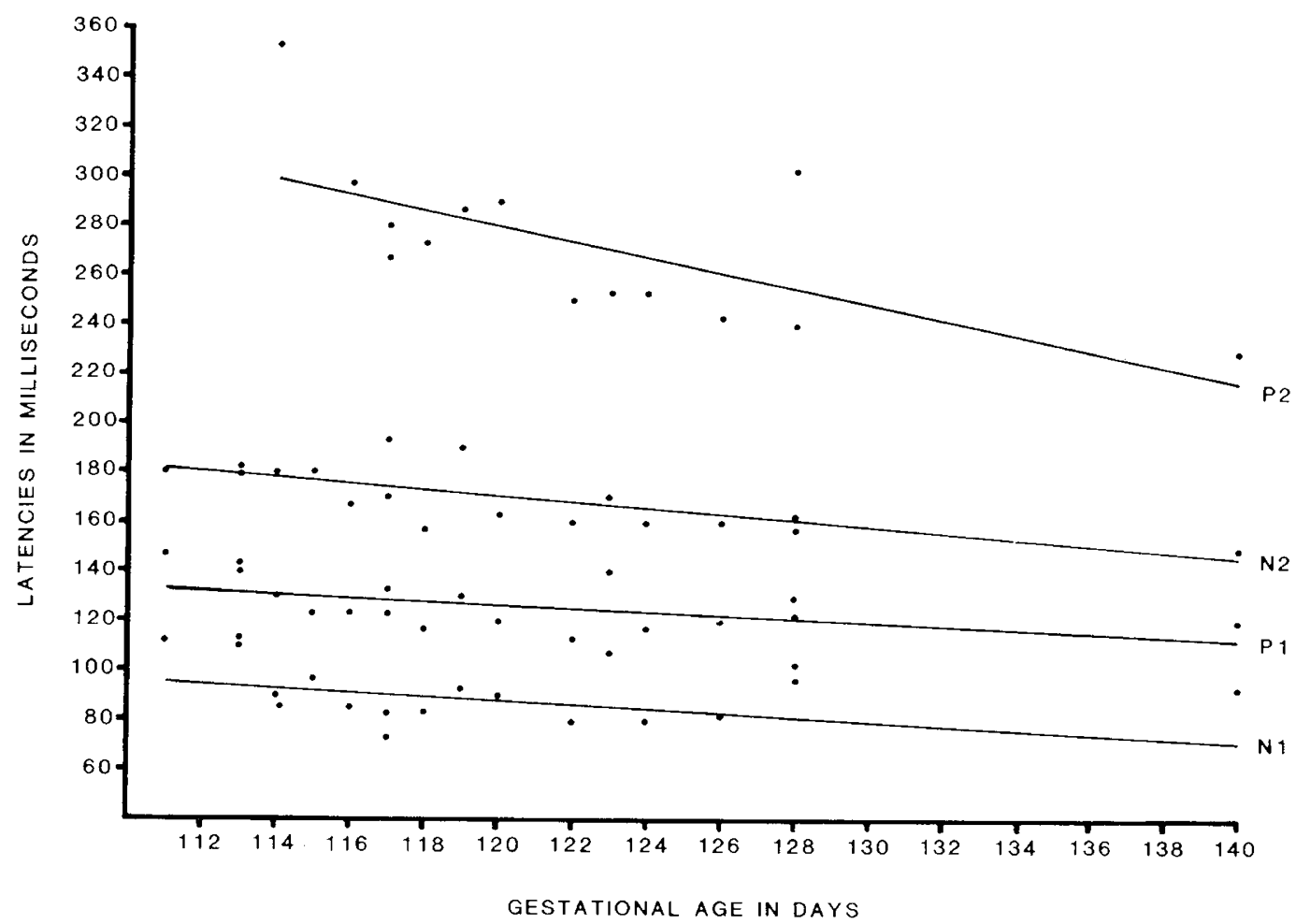

Fig. 5. Correlation of latency values of VEP components $N_{1}, P_{1}, N_{2}$, and $P_{2}$ from five fetal lambs, with gestational age.

Table 1. VEP latency measurements from five preterm fetal lambs at 111-120 days and 121-128 days gestation (mean \pm SEM)

\begin{tabular}{ccccc}
\hline & \multicolumn{4}{c}{ Fetal latencies in MS } \\
\cline { 2 - 5 } Days & $\mathrm{N}_{1}$ & $\mathrm{P}_{1}$ & $\mathrm{~N}_{2}$ & $\mathrm{P}_{2}$ \\
\hline $111-120$ & $93.9 \pm 2.4$ & $130.0 \pm 2.1$ & $176.4 \pm 2.5$ & $292.4 \pm 7.0$ \\
& $(33)^{*}$ & $(33)$ & $(32)$ & $(21)$ \\
$121-128$ & $91.4 \pm 3.1$ & $123.9 \pm 2.4$ & $161.7 \pm 1.5$ & $256.7 \pm 6.1$ \\
& $(18)$ & $(18)$ & $(18)$ & $(18)$ \\
\hline
\end{tabular}

* No. of recording runs.

neuroelectric signal. In addition, when the fetus is serially tested from 111 days gestation, the VEP can be used to record changes in fetal visual responsiveness during the last 30 days of pregnancy. The latency changes presented in this study may be indicative of maturational processes occurring within the visual system of the fetal lamb. From histopathological assessment of the cornea, this method of VEP testing does not appear to cause serious injury to the tested eye.

The initial recordings of fetal VEPs between 111-114 days gestation exhibit the $N_{1}, P_{1}$, and $N_{2}$ wave components. One fetus (Fig. 4) showed no VEP components of any kind at 112 days but displayed $N_{1}, P_{1}$, and $N_{2}$ components by 114 days. The presence of these components at this stage of gestation indicates that certain visual structures are functional. Initial onset of function of these structures may occur earlier in fetal development than 111-114 days gestation used in this study. Another possible explanation is the fetus may be recovering from the surgical stress of instrumentation. These issues will be resolved in further studies by surgically instrumenting fetal lambs at an earlier gestational age. Initial VEPs obtained from 111-114 days did not contain the $\mathrm{P}_{2}$ component. This later component of the fetal VEP, however, was measureable by 115-118 days.

Although the generators of the visual evoked potential have not been clearly defined, the early wave components, $N_{1}, P_{1}$, and
$\mathrm{N}_{2}$ are presumed to represent the neuroelectric activity along the optic nerve, optic chiasm, and the lateral geniculate body, respectively $(8,9)$. The later component, $P_{2}$, presumably represents the spread of the neuroelectric signal as it reaches and passes into the cortical tissue at the occipital lobe. The present data suggest that development of the optic tracts, optic chiasm, and the lateral geniculate body is nearly complete when the initial VEP recordings were made from 111-114 days. The sudden appearance of the $\mathrm{P}_{2}$ component from 115-118 days gestation and latency change from 118-128 days may be indicative of increased synaptogenesis and myelination within the cortical tissue of the occipital lobe $(8,10)$.

Auditory development is also known to be occurring in the fetal lamb from 117 days gestation $(11,12)$. In these studies, auditory brainstem responses were generated by click stimuli from a small earphone secured in the right ear of the fetal lamb and recorded from subdermal stainless steel scalp electrodes. Onset of auditory function appears to take place at approximately 117 days gestation, since auditory brainstem responses were first recorded after 117 days, despite testing from 110-116 days. As the fetal lamb becomes older, peak latencies of the auditory responses rapidly decrease $(11,12)$.

In utero assessment of maturational sequences within the visual system of the fetal lamb is now possible using the techniques described in this study. Changes in latency values from fetal lamb VEP indicate that maturation within this sensory system occurs during the final weeks of fetal life. These findings have application in fetal neurotoxicology since the immature central nervous system is most vulnerable to toxic damage when growth is rapid (13). Furthermore, neurological damage from a perinatal insult may not be evident until days or weeks after the event (14). Since longitudinal testing of the visual system of the fetus can be conducted using the described techniques in this study, this animal model has application for studying the effects of adverse perinatal events and drugs on neurological development during fetal growth. 
Acknowledgments. The authors thank Dr. Alan Salamy and the Infant Hearing Assessment Foundation, San Francisco, CA, for providing the Synap I Signal Averaging Computer used in this study.

\section{REFERENCES}

1. Gambi D, Rossini PM, Albertini G, Sollazzo D, Torrioli MG, Polidori GC 1980 Follow-up of visual evoked potential in full-term and pre-term control newborns and in subjects who suffered from perinatal respiratory distress. Electroencephalogr Clin Neurophysiol 48:509-516

2. Hrbek A, Karlberg P, Olsson T 1973 Development of visual and somatosensory evoked responses in pre-term newborn infants. Electroencephalogr Clin Neurophysiol 34:225-232

3. Barnet AB, Friedman SL, Weiss IP, Ohlrich ES, Shanks B, Lodge A 1980 VEP development in infancy and early childhood. A longitudinal study. Electroencephalogr Clin Neurophysiol 49:476-489

4. Blom JL Barth PG, Visser SL 1980 The visual evoked potential in the first six years of life. Electroencephalogr Clin Neurophysiol 48:395-405

5. Ferriss GS, Davis GD, Dorsen MMcF, Hackett ER 1967 Changes in latency and form of the photically induced average evoked response in human infants. Electroencephalogr Clin Neurophysiol 22:305-312

6. Woods JR Jr, Coppes V, Brooks DE, Knowles PJ, Freeman M, Parisi V,
Omara P, McCarty GE 1981 Birth asphyxia. I. Measurement of visual evoked potential (VEP) in the healthy fetus and newborn lamb. Pediatr Res 15:14291432

7. Woods JR Jr, Parisi V, Coppes V, Brooks DE 1983 Maturational sequence of the visual system: serial measurements of visual evoked potential and electroretinogram in the healthy neonatal lamb. Am J Obstet Gynecol 145:738743

8. Kayama Y 1974 Evoked potentials of the central visual system during and after hypoxia in cats. Electroencephalogr Clin Neurophysiol 36:619-628

9. Kooi KA, Marshall RE 1979 Anatomic and electrophysiologic substrates of the visual evoked potential. In: Kooi KA, Marshall RE (ed) Visual Evoked Potentials in Central Disorders of the Visual System. Harper and Row, Baltimore, pp 7-18

10. Barlow RM 1969 The foetal sheep: morphogenesis of the nervous system and histochemical aspects of myelination. J Comp Neurol 135:249-262

11. Woods JR Jr, Plessinger MA, Mack CE 1984 Fetal auditory brainstem evoked response (ABR). Pediatr Res 18:83-85

12. Woods JR Jr, Plessinger MA 1985 The fetal auditory brainstem response: serial measurements at two stimulus intensities. Otolaryngol Head Neck Surg 93:759-764

13. Mattsson JL, Fry WN, Boward CA, Miller E 1978 Maturation of the visual evoked response in newborn miniature pigs. Am $\boldsymbol{J}$ Vet Res 39:1279-1281

14. Bronshvag MM 1978 Cellular basis of anoxic-ischemic brain injury. West $\mathbf{J}$ Med 129:8-18 